\title{
The Effect of Epigallocatechin Gallate on Intestinal Motility in Mice
}

\author{
Michal CEREGRZYN and Atsukazu KUWAHARA \\ Laboratory of Physiology, Institute for Environmental Sciences, University of Shizuoka, Shizuoka, Japan
}

\begin{abstract}
Objectives: The epigallocatechin-3-gallate (EGCg) that is present in human diet originates mainly from tea leaves. Catechins have a number of possible application as medicines, however, there is no consistent evidence showing their influence on the gastrointestinal tract. Thus, the aim of the present study was to investigate the effect of EGCg on the motility of the murine isolated intestine.

Methods: Segments of jejunum submerged in Krebs buffer were exposed to EGCg and the response was recorded under isometric conditions.

Results: EGCg induced a dose-dependent inhibition of spontaneous activity in the jejunum. EGCg induced a decrease in the amplitude and frequency of jejunal contractions. Moreover, the rythmicity of spontaneous activity was altered in the presence of EGCg. A significant effect of EGCg was observed in the presence of $10^{-4} \mathrm{M}$. The effect of EGCg was in part inhibited by pretreatment with methylene blue (guanylate cyclase inhibitor), while tetrodotoxin, (sodium channel blocker), L-nitro arginine methyl ester (nitric oxide synthase inhibitor), and N-ethylmaleimide (adenylate cyclase inhibitor) showed no effect.

Conclusions: The results of the present study suggest that EGCg inhibits the motility of the jejunum by direct action on smooth muscle cells where a guanylate cyclase-dependent mechanism may be partly involved.
\end{abstract}

Key words: epigallocatechin, intestine, motility, mouse

\section{Objectives}

Catechins originate from different plants. They are easily absorbed in the gastrointestinal tract from ingested cocoa (1), green tea (2), or black tea (3). Tea is the main source of catechins in humans. The principal catechins present in green tea include epicatechin, epicatechin-3-gallate, epigallocatechin, and epigallocatechin-3-gallate (EGCg).

Catechins influence various gastrointestinal functions, including gastrointestinal motility and transit. An early study reported that gossypin, epicatechin and hydroxyethyl rutosides delayed intestinal transit in a dose-dependent manner in mice. Naloxone, yohimbine, and phentolamine antagonized the effects of these compounds. Prazosin, propranolol, atropine, physostigmine, hexamethonium, pheniramine, and metiamide had no effect. Thus, it has been suggested that opiate and alpha-2 adrenergic receptors mediate the flavonoid-induced delay in the

Received Aug. 29 2002/Accepted Jan. 312003

Reprint requests to: Michal CEREGRZYN

Laboratory of Physiology, Institute for Environmental Sciences, University of Shizuoka, 52-1 Yada, Shizuoka, Shizuoka 422-8526, Japan

TEL/FAX: +81(54)264-5794

E-mail: gp1163@mail.e.u-shizuoka-ken.ac.jp transit in the small intestine (4). Another study concerning the effects of polyphenolic compounds on gastrointestinal transit has shown that kampferol morin, myricetin, and rutin have a strong, dose-dependent inhibitory effect on charcoal transit in mice. Catechin, however, did not show a significant effect (5). An in vitro study has shown that EGCg has direct depolarizing effects on a population of myenteric neurons in the guinea-pig small intestine (6). Moreover, EGCg facilitates cholinergic ganglion transmission in enteric neurons (7).

Since catechins are compounds of potential use as medicines, there is a need to investigate their effects on various organs. Based on available evidence it is unclear if catechins are potent factors influencing gastrointestinal motility. In addition, there is no study reporting the effects and mechanisms of catechins on the functional activity of the intestine in vitro. Thus, the aim of the present study was to characterize the effects and mechanisms of action of EGCg on mechanical activity of the mouse small intestine.

\section{Methods}

Animals

Female, ddy mice $(31.7 \pm 6.7 \mathrm{~g})$ were used in all experi- 
ments. The animals had free access to laboratory chow and tap water before the experiment and were kept under artificial lighting 12 hours per day at $20^{\circ} \mathrm{C}$ to $22^{\circ} \mathrm{C}$. Experimental protocols were carried out according to the principles and guidelines of the National Institute for Physiological Sciences in Japan.

\section{In vitro intestinal motility recording}

The animals were anesthetized with ether and then stomach and intestinal tissues were harvested. Segments of proximal jejunum (approximately $7 \mathrm{~mm}$ long) were prepared and mounted in a 10-ml organ bath. The organ bath contained Krebs solution with the following composition (in $\mathrm{mM}$ ): $\mathrm{NaCl} \mathrm{118,} \mathrm{KCl} 4.8$, $\mathrm{KH}_{2} \mathrm{PO}_{4} 1.2, \mathrm{MgSO}_{4} 1.2, \mathrm{CaCl}_{2} 1.9, \mathrm{NaHCO}_{3} 25$, and glucose 10.1. The Krebs solution was constantly gassed with a $95 \% \mathrm{O}_{2}$, $5 \% \mathrm{CO}_{2}$ mixture. The temperature of the buffer was maintained at $37^{\circ} \mathrm{C}$. Changes in the tension of the strips were recorded isometrically with force transducers (type 45196A, NEC San-ei Instruments Ltd., Japan) coupled with a PowerLab recording system (ADInstruments, Australia).

The mechanical activity of jejunal segments was recorded in accordance with the following schedule. The strips were allowed to equilibrate for 30 minutes. Next, acetylcholine (ACh, $10^{-6} \mathrm{M}$ ) was added to the bath. Next EGCg was added to the chamber in a noncumulative manner in concentrations $10^{-6}$, $10^{-5}, 10^{-4}$, and $10^{-3} \mathrm{M}$ (Fig. 1). For the quantitative analysis, the following concentrations were used $3.7 \cdot 10^{-5}, 1.1 \cdot 10^{-4}, 3.3 \cdot 10^{-4}$, and $1 \cdot 10^{-3} \mathrm{M}$. The response to each administration of catechins was recorded for 10 minutes. Then the chamber was washed out for the next 10 minutes, followed by the administration of EGCg. At the end of the experiment, another ACh trial was performed in the same manner as at the beginning of the experiment.

\section{Mechanism of catechin action}

After equilibration, EGCg was added in a concentration of $5 \cdot 10^{-4} \mathrm{M}$. Next, compounds of known pharmacological action were added to the chamber, and in their presence the segments

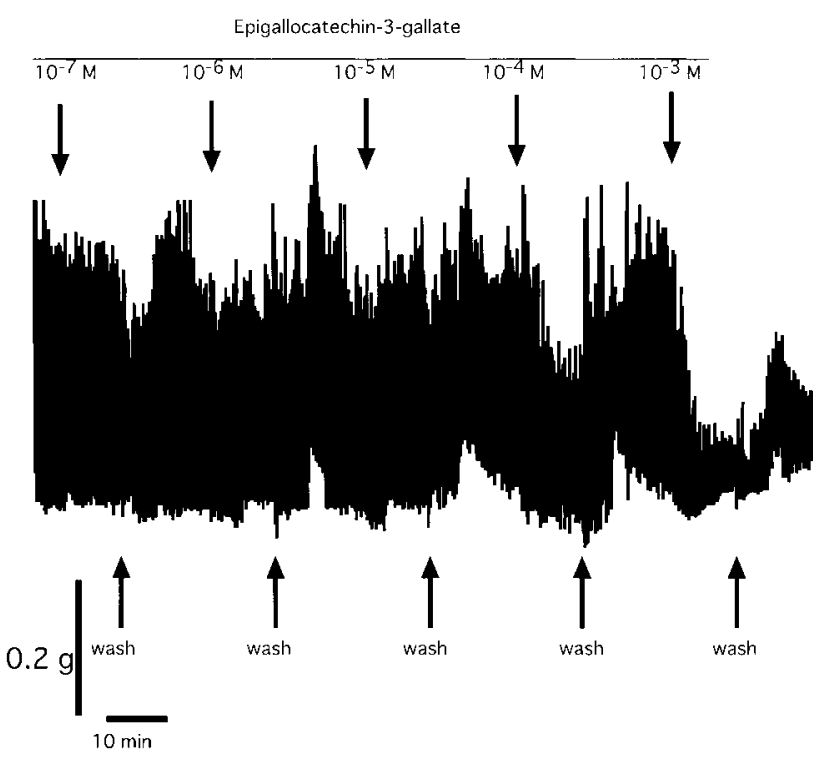

Fig. 1 The effect of EGCg on spontaneous contractions of the mouse jejunum in vitro. Representative tracing of isometric recording. The changes in tension are expressed in grams (g). were again exposed to EGCg. The following compounds were used: $10^{-6} \mathrm{M}$ tetrodotoxin (TTX, sodium channel blocker), $10^{-4} \mathrm{M}$ methylene blue (MB, guanylate cyclase inhibitor), $10^{-4} \mathrm{M}$ L-nitro arginine methyl ester (L-NAME, nitric oxide synthase inhibitor), and $10^{-5} \mathrm{M}$ N-ethylmaleimide (NEM, adenylate cyclase inhibitor).

\section{Chemicals}

EGCg was a gift from Tokyo Food Techno Co. LTD. (Tokyo, Japan). The substance was -(-) EGCg isomer. The purity of the compound was $98.4 \%$. EGCg was isolated from green tea leaves using the modified method presented by Hara et al. (8). EGCg was isolated from green tea leaves using the following extraction: first, hot water extract, then organic solvent extract (ethyl acetate). The next step was a crude isolation using synthetic resin (Sepabeds, Mitsubishi Chemical Company, Japan) followed by an isolation using ODS (Siliciferous with octa decyl silyl). Finally, EGCg was crystallized from methanolwater solution. The rest of the compounds were purchased from Sigma (St. Louis, USA).

\section{Statistics}

All values are expressed as means \pm SEM. Results from control- and EGCg-treated tissues were compared by ANOVA and Tukey post hoc test. A p value $<0.05$ was considered to be statistically significant.

\section{Results}

\section{The effect of catechins on spontaneous contractions}

EGCg induced a decrease in the spontaneous activity of the jejunum. The effect was observed as a decrease in the frequency and amplitude of contractions (Fig. 1, 2, and 3). The decrease in amplitude induced by EGCg was dose-dependent, while the highest concentration EGCg induced a significant increase in the frequency of contractions (Fig. 3). In the presence of $\mathrm{EGCg}$, the rythmicity of spontaneous contractions was markedly altered (Fig. 2). The effect of EGCg $3.3 \times 10^{-4} \mathrm{M}$ lasted approximately 20 minutes. It was necessary to change the buffer twice to restore the spontaneous contractility close to the initial level.

\section{Acetylcholine-induced contractions}

ACh was used as a control for the contractility of the intestine at the beginning and the end of the experiment. The addition of $10^{-6} \mathrm{M}$ of $\mathrm{ACh}$ induced a single, strong contraction that lasted approximately 30 seconds. Then the activity of the intestine returned to the control value. There were no significant differences between the control contraction at the beginning of the experiment and that induced at the end (Fig. 4).

\section{The mechanism of catechin action}

In the present study TTX, $10^{-6} \mathrm{M}, \mathrm{NEM}, 10^{-5} \mathrm{M}$, and LNAME, $10^{-4} \mathrm{M}$ did not significantly affect the spontaneous activity of the isolated intestine. Moreover, they had no significant effects on an EGCg-induced decrease in spontaneous jejunal activity, both in terms of the frequency and amplitude of the contractions. On the other hand, guanylate cyclase 


\section{Control

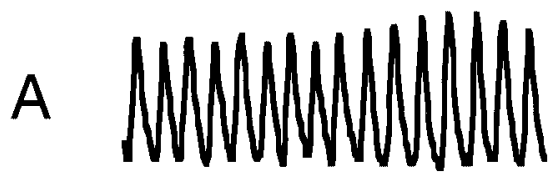

B EGcG $3.3 \times 10.4 \mathrm{M}$

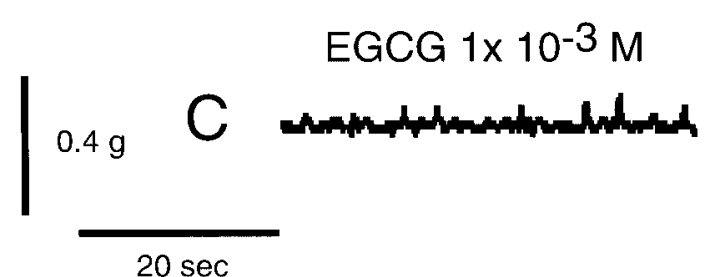

Fig. 2 The effect of EGCg on spontaneous contractions of the mouse jejunum in vitro. Representative tracing of control activity, before the addition of EGCg (A), activity 10 minutes after the addition of $3.3 \cdot 10^{-4} \mathrm{M}(\mathrm{B})$ and $1 \cdot 10^{-3} \mathrm{M} \mathrm{EGCg} \mathrm{(C).}$
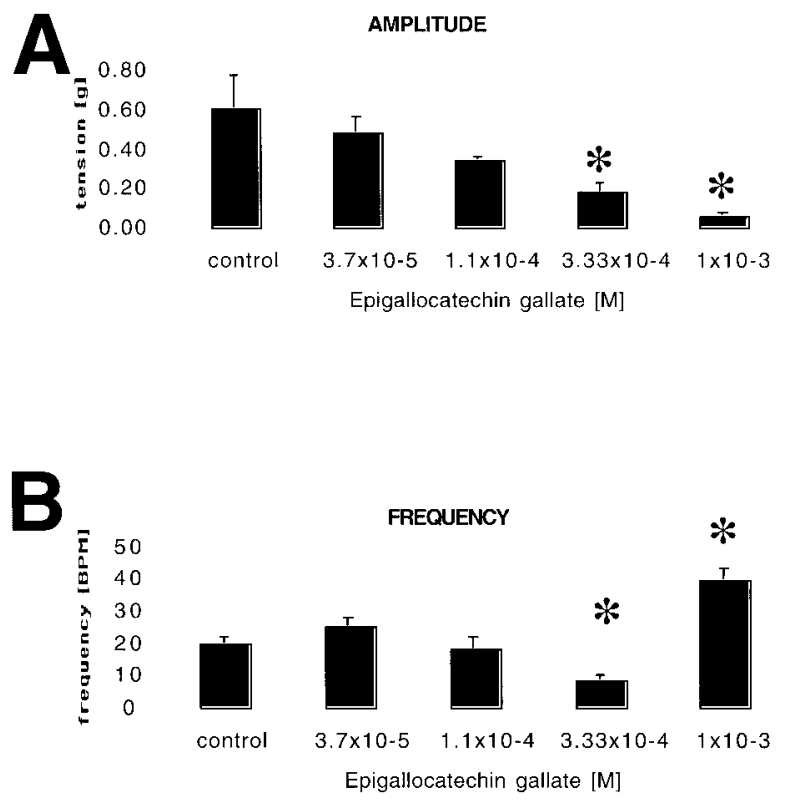

Fig. 3 The effect of EGCg on the amplitude (A) and frequency (B) of spontaneous contractions of the mouse jejunum in vitro. The amplitude is expressed as the change in the tension of strips in grams and the frequency is expressed as the number of events (beats) per minute (BPM). Values are expressed as means \pm SEM $(n=6)$. Significantly different from the control group * $P<0.05$ (ANOVA, Tukey post hoc test).

inhibitor MB $10^{-4} \mathrm{M}$ (which also showed no significant influence on spontaneous motility) effectively influenced the EGCginduced decrease in the amplitude of jejunal motility. However, MB did not completely reverse the EGCg-induced inhibition of spontaneous motility. Moreover, MB showed no significant
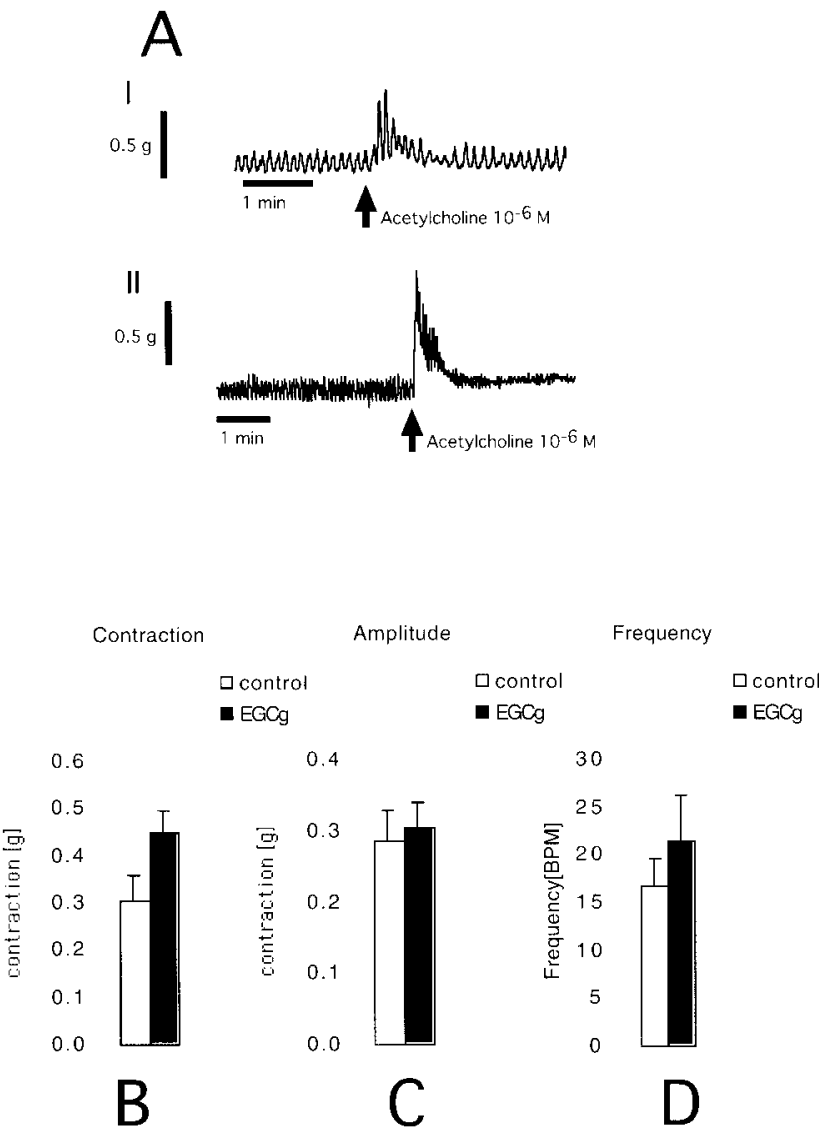

Fig. 4 The effect of acetylcholine on the tension of the mouse intestine. Representative tracing of control (I) and after EGCg $5 \cdot 10^{-4} \mathrm{M}$ (II) responses (A). Comparison between the control group (white) and post-EGCg group (black). The maximal contraction (B), amplitude (C), and frequency (D) recorded after $\mathrm{ACh}$ administration are expressed as means $\pm \operatorname{SEM}, n=6$. There is no significant difference between the groups (Student's $t$ test).

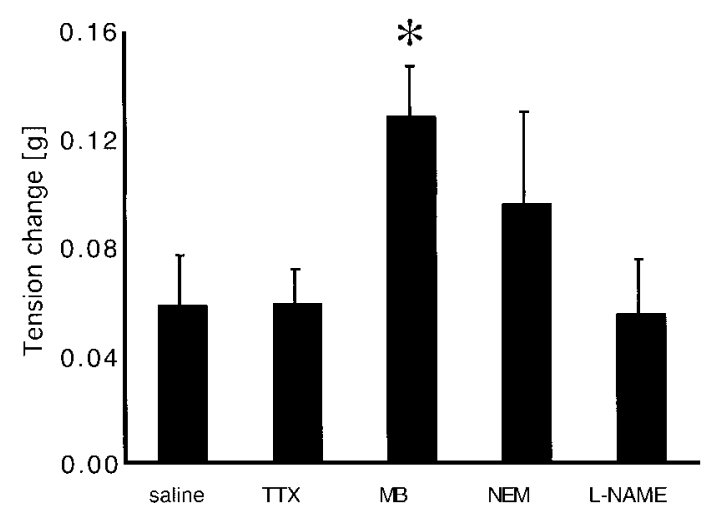

Fig. 5 The effects of various antagonists on EGCg $\left(5 \cdot 10^{-4} \mathrm{M}\right)$ induced supression in intestinal motility in vitro. Data show the amplitude of contractions expressed as the change in the tension of strips in grams measured 10 minutes after EGCg administration. The concentrations of compounds were: TTX-tetrodotoxin $10^{-6} \mathrm{M}$, $\mathrm{MB}-$ methylene blue $10^{-4} \mathrm{M}$, NEM-N-ethylmaleimide $10^{-5} \mathrm{M}$, L-NAME-L-nitro arginine methyl ester $10^{-4} \mathrm{M}$. Values are expressed as means \pm SEM $(n=6)$. Significantly different from the saline group * $\mathbf{P}<0.05$ (Student's unpaired $\mathbf{t}$ test).

influence on the frequency of contractions. (Figs. 5 and 6). 


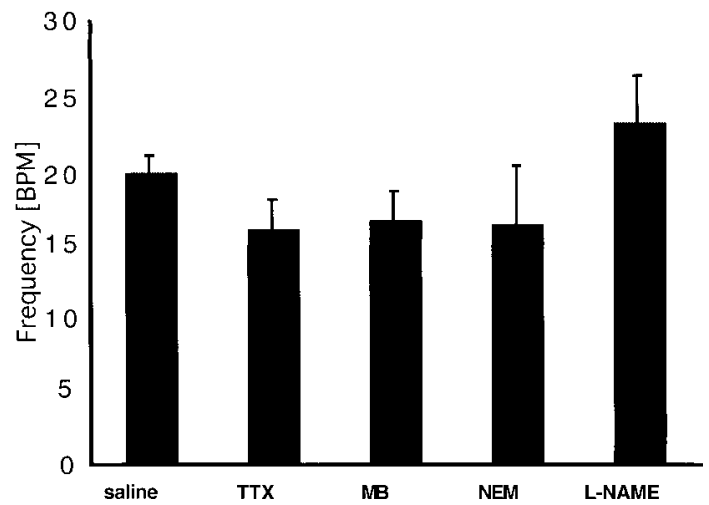

Fig. 6 The effect of various antagonists on EGCg $\left(5 \cdot 10^{-4} \mathrm{M}\right)$ induced supression in intestinal motility in vitro. Data show the frequency (contractions/minute) 10 minutes after EGCg administration. The concentrations of compounds were: TTX-tetrodotoxin $10^{-6} \mathrm{M}$, MB - methylene blue $10^{-4} \mathrm{M}, \mathrm{NEM}-\mathrm{N}$-ethylmaleimide $10^{-5} \mathrm{M}$, L-NAME-L-nitro arginine methyl ester $10^{-4} \mathrm{M}$ on the frequency of spontaneous contractions of the mouse jejunum in vitro. Values are expressed as means $\pm \operatorname{SEM}(n=6)$.

\section{Conclusions}

EGCg performs a variety of biological activities, including antitumor as well as antimicrobial activity against some pathogens. There is evidence suggesting that the treatment of mouse skin with EGCg prevents UV-B-induced leukocyte infiltration, antigen-presenting cell infiltration, and oxidative stress. In this way EGCg may evoke the prevention of UV-B-induced immunosuppression and photocarcinogenesis (9). Moreover, pretreatment with EGCg restores the UV-induced decrease in the total glutathione level and affords protection to the antioxidant enzyme glutathione peroxidase (9). EGCg shows the strongest activity of the six tea catechins tested against Helicobacter pylori (10). EGCg selectively up-regulates the production of IL-12 and tumor necrosis factor alpha, and down-regulates IL-10 production in macrophages induced by Legionella pneumophila infection (11).

Catechins influence gastrointestinal functions other than motility. Meciadanol (O-methyl-3(+)-catechin), a synthetic flavonoid, protects against ulcerogens and does not affect either gastric acid secretion or pepsin output in rats $(12,13)$. On the other hand, catechins inhibit gastric $\mathrm{H}+, \mathrm{K}(+)$-ATPase activity, and EGCg is the most potent inhibitor. The intensity of inhibitor activity parallels the number of phenolic hydroxy groups in the molecule. These findings suggest that the anti-secretory and anti-ulcerogenic effects of catechins are due to their inhibitory activity on gastric $\mathrm{H}+, \mathrm{K}(+)$-ATPase (14).

The findings of the present study showed that EGCg derived from tea leaves can alter spontaneous activity of the small intestine. However, the effect appears at relatively high concentrations. The level of availability of catechins has been confirmed at lower concentrations $(15,3)$. The plasma concentrations and doses presented in recent studies were much lower than that presented as effective in the present study. The lowest concentration that was effective in the present study was $10^{-4} \mathrm{M}$ of EGCg. It was reported that in brews the extraction efficiency of catechins is approximately $50 \%$ (16). Based on the catechin content in tea leaves, it can be calculated that the concentration of EGCg in a brew is $74.5 \mathrm{mg} / 100 \mathrm{ml}$, which gives a molar concentration $1.6510^{-3} \mathrm{M}$. Thus, it is possible that tea drinking can influence intestinal motility. On the other hand, the proposed antioxidative action that has been reported at the concentrations in the range of $10^{-6} \mathrm{M}(2,17)$ may be reached without a disturbance of gastrointestinal motility. However, the potential inhibitory influence of catechins on intestinal motility is possible in the case of longer exposures or higher concentrations.

The present study shows that the frequency of contractions in the presence of the highest concentration of EGCg increased, while the amplitude was significantly smaller than that of the control. Such an effect was a result of the presence of a large number of small irregular contractions. This activity was still higher than the noise that was visible in the presence of papaverine $10^{-3} \mathrm{M}$. In the presence of papaverine, the amplitude and frequency was completely abolished (data not shown). Thus, one can conclude that the activity observed in the presence of EGCg was the actvity of intestinal smooth muscle. Moreover, the numbe of small contractions suggests that in the presence of EGCg the integrated activity of the intestinal segment is markedly disrupted. Smooth muscle in the intestine works as an integrated mechanism. Contractions are evoked by changes in the membrane potential, called slow waves, that originate from pacemaker regions located at the myenteric and submucosal borders of circular muscle (18). The slow waves propagate rapidly in the muscle. Slow wave transmition is facilitated by the peripheral network of intersitial cells, the abundance of gap junctions, and the neuronal network of the enteric nervous system. It leads to organized contractions in the whole segmnent of the intestine. Thus, the decrease in amplitude with an increase in frequency suggests that EGCg disrupts the organized contractions in the intestinal segments.

The present study shows that EGCg induces a significant disruption of spontaneous activity in the small intestine. The mechanism of this action most likely involves direct cellular action since TTX and L-NAME were ineffective in preventing the inhibiting effect of EGCg. The effect of MB suggests that, in part, spontaneous motility inhibition is induced by the activation of guanylate cyclase, while adenylate cyclase is not involved in this action. The precise mechanism of this effect remains to be investigated. The present study shows that EGCg effects (myenteric neuron depolarization) observed at the cellular level at concentrations in the range of $10^{-6} \mathrm{M}(6,7)$ do not induce significant effects at the tissue level. It is possible that in the experimental design of the present study, local concentrations were smaller than the general concentration in the chamber. However, the lack of the TTX effect suggests the direct action of EGCg on intestinal smooth muscle. It was reported that catechins directly activate superoxide dismutase in the rat brain (19). Moreover, catechins inhibit xanthine oxidase in liver (20). Thus, it is possible that EGCg induces a non-selective effect on intracellular functions that results in the inhibition of spontaneous motility in the intestine. 


\section{References}

( 1 ) Baba S, Osakabe N, Yasuda A, Natsume M, Takizawa T, Nakamura T, Terao J. Bioavailability of (-)-epicatechin upon intake of chocolate and cocoa in human volunteers. Free Radic. Res. 2000; 33: 635-641.

( 2 ) Miyazawa T. Absorption, metabolism and antioxidative effects of tea catechin in humans. Biofactors 2000; 13: 55-59.

(3) Warden BA, Smith LS, Beecher GR, Balentine DA, Clevidence BA. Catechins Are Bioavailable in Men and Women Drinking Black Tea throughout the Day. J. Nutr. 2001; 131: 1731-1737.

(4) Viswanathan S, Thirugnana Sambantham P, Bapna JS, Kameswaran L. Flavonoid-induced delay in the small intestinal transit: possible mechanism of action. Arch. Int. Pharmacodyn. Ther. 1984; 270: 151-157.

( 5 ) Di Carlo G, Autore G, Izzo AA, Maiolino P, Mascolo N, Viola P, Diurno MV, Capasso F. Inhibition of intestinal motility and secretion by flavonoids in mice and rats: structure-activity relationships. J. Pharm. Pharmacol. 1993; 45: 1054-1059.

( 6 ) Homma T, Hirai K, Hara Y, Katayama Y. Tea catechin, (-)epigallocatechin gallate, causes membrane depolarizations of myenteric neurons in the guinea-pig small intestine. Neurosci. Lett. 2001; 309: 93-96.

( 7 ) Katayama Y, Homma T, Hara Y, Hirai K. Tea catechin, (-)epigallocatechin gallate, facilitates cholinergic ganglion transmission in the myenteric plexus of the guinea-pig small intestine. Neurosci. Lett. 2002; 319: 63-66.

( 8 ) Hara Y, Matsuzaki S, Nakamura K. Anti-tumor activity of tea catechins. J. Agr. Chem. Soc. Jap. 1985; 59: 129-134.

(9) Katiyar SK, Afaq F, Perez A, Mukhtar H. Green tea polyphenol (-)-epigallocatechin-3-gallate treatment of human skin inhibits ultraviolet radiation-induced oxidative stress. Carcinogenesis 2001; 22: 287-294.

(10) Mabe K, Yamada M, Oguni I, Takahashi T. In vitro and in vivo activities of tea catechins against Helicobacter pylori. Antimicrob. Agents. Chemother. 1999; 43: 1788-1791.

(11) Matsunaga K, Klein TW, Friedman H, Yamamoto Y. Legionella pneumophila Replication in Macrophages Inhibited by Selective Immunomodulatory Effects on Cytokine Formation by
Epigallocatechin Gallate, a Major Form of Tea Catechins. Infect. Immun. 2001; 69: 3947-3953.

(12) Konturek SJ, Kitler ME, Brzozowski T, Radecki T. Gastric protection by meciadanol. A new synthetic flavonoid inhibiting histidine decarboxylase. Dig. Dis. Sci. 1986; 31: 847852.

(13) Jayaraj AP, Lewin MR, Tovey FI, Kitler ME, Clark CG. The protective effect of Meciadanol (O-methyl-3(+)-catechin) on experimental ulceration. Eur. J. Pharmacol. 1988; 147: 265271.

(14) Murakami S, Muramatsu M, Otomo S. Gastric H+, K(+)ATPase inhibition by catechins. J. Pharm. Pharmacol. 1992; 44: 926-928.

(15) Chow HH, Cai Y, Alberts DS, Hakim I, Dorr R, Shahi F, Crowell JA, Yang CS, Hara Y. Phase I pharmacokinetic study of tea polyphenols following single-dose administration of epigallocatechin gallate and polyphenon E. Cancer Epidemiol. Biomarkers Prev. 2001; 10: 53-58.

(16) Lakenbrink C, Lapczynski S, Maiwald B, Engelhardt UH. Flavonoids and other polyphenols in consumer brews of tea and other caffeinated beverages. J. Agric. Food. Chem. 2000; 48: 2848-2852.

(17) Anderson RF, Fisher LJ, Hara Y, Harris T, Mak WB, Melton LD, Packer JE. Green tea catechins partially protect DNA from (.)OH radical-induced strand breaks and base damage through fast chemical repair of DNA radicals. Carcinogenesis 2001; 22: 1189-1193.

(18) Makhlouf GM, Smooth muscle of the gut, in Textbook of gastroenterology, M. Yamada, Editor. 1999, Lippincott Williams \& Wilkins: Philadelphia. p. 82-105ß.

(19) Komatsu M, Hiramatsu M. The efficacy of an antioxidant cocktail on lipid peroxide level and superoxide dismutase activity in aged rat brain and DNA damage in iron-induced epileptogenic foci. Toxicology 2000; 148: 143-148.

(20) Aucamp J, Gaspar A, Hara Y, Apostolides Z. Inhibition of xanthine oxidase by catechins from tea (Camellia sinensis). Anticancer. Res. 1997; 17: 4381-4385. 